\title{
QUI'M DISSES, NON A DOS ANS DI GUIRAUT RIQUIER E LA SUCCESSIONE DI ALFONSO X
}

\author{
Fabio BARBERINI \\ fbobarb@gmail.com \\ CNRS / Université de Toulouse "Jean Jaurès"
}

Decimas tuas et primitias tuas non tardabis reddere: primogenitum filiorum tuorum dabis mihi (Exodus, XXII, 23)

1. Il decimo vers di Guiraut Riquier (= GrRiq), Qui $\cdot$ m disses, non a dos ans ${ }^{1}$, fu composto, secondo la rubrica del Libre, nel settembre del $1276^{2}$. La lettura vulgata si limita a segnalare che questo vers commenta i problemi relativi alla successione al trono di Castiglia-León. Per Anglade «le sirventes de Riquier ... est comme un écho des murmures et des critiques qu'il entendait autour de lui» ${ }^{3}$. Per Riquer il trovatore narbonese

demuestra aquí un evidente disgusto y critica, sobre todo en las tornadas, a Alfonso X. Es esta pieza una curiosa muestra de la franqueza y valentía con que algunos trovadores ... opinaban sobre la política y actos de reyes y grandes señores en composiciones escritas para ser divulgadas en sus propias cortes ${ }^{4}$.

${ }^{1}$ Faccio riferimento all'edizione di Monica Longobardi, «I vers del trovatore Guiraut Riquier», Studi Mediolatini e Volgari, XXIX (1982-1983), pp. 17-163 [75]. Il testo del componimento è riprodotto in Appendice. Sul Libre di GrRiq cfr. Valeria Bertolucci Pizzorusso, «Il canzoniere di un trovatore: il "libro" di Guiraut Riquier» e «Libri e canzonieri d'autore nel Medioevo: prospettive di ricerca», in Ead., Morfologie del testo medievale, Bologna, Il Mulino, 1989, rispettivamente pp. 87-124 (con edizione e commento del rubricario riquieriano) e 125145 [contributi originariamente pubblicati in rivista nel 1978 e nel 1984]; Ead., «Un progetto di edizione del "Libre" di Guiraut Riquier e altre osservazioni», Tenso, 9 (1994), pp. 106-125 e Ead., «Per una nuova edizione del libre di Guiraut Riquier. Con una nota sulle etimologie riquieriane», Lecturae tropatorum, 8 (2015), pp. 1-14 (in rete all'indirizzo $<$ http://www.lt.unina. it/BertolucciPizzorusso-2015S.pdf>).

${ }^{2}$ Imprecisa la cronologia di Gerardo Larghi, s.v. «Guiraut Riquier», in Saverio Guida e Gerardo Larghi, Dizionario Biografico dei Trovatori, Modena, Mucchi editore, 2014, pp. 291296 [294]: «tra il 1275 e il 1280, ideò Jhesus Cristz, filh de Dieu viu (BdT 248.46), Karitatz ez amors e fe (BdT 248.48), Xristias vei perilhar (BdT 248.87), D'Astarac venia (BdT 248.22) e, nel dicembre dello stesso anno, Qui.m disses, non a dos ans (BdT 248.68)»; se il «dicembre dello stesso anno» si riferisce al 1280 -estremo del segmento cronologico indicato all'inizio dell'enunciato- l'indicazione è due volte errata, per il mese (che, in accordo con la rubrica, deve essere settembre) e per l'anno (che deve essere il 1276).

${ }^{3}$ Joseph Anglade, Le troubadour Guiraut Riquier. Étude sur la décadence de l'ancienne poésie provençale, Bordeaux, Paris, Feret et Fils éditerus, Albert Fontemoing, 1909, p. 157.

${ }^{4}$ Martín de Riquer, Los trovadores. Historia literaria y textos, 3 vols., Barcelona, Editorial 
Se si considera però che GrRiq parla dall'interno della corte (e della corte riflette i malumori) ${ }^{5}$, tali interpretazioni lasciano nell'ombra un punto essenziale, vale a dire: qual è la posizione di GrRiq nei confronti dei due pretendenti al trono? E, soprattutto, qual è il comportamento di Alfonso X che ha provocato tante critiche?

Se interpreto correttamente il pensiero di quanti si sono occupati di Qui.m disses $^{6}$, mi sembra di capire che i rimproveri che il trovatore rivolge al Re derivino dal fatto che, dopo la morte dell'Infante D. Fernando de la Cerda (1275), Alfonso X si sia schierato dalla parte del figlio (D. Sancho) contro il legittimo diritto dell'omonimo nipote (D. Alfonso de la Cerda); circostanza che avrebbe provocato più d'un malumore nell'entourage del Rei Sabio. Questa, almeno, la conclusione che mi sembra possibile inferire dall'interpretazione proposta per i vv. 19-21:

\section{Mala veyra sos efans, si 1 pus de la gent ver ditz que vius n'er despostaditz!}

1) Manuel Milà y Fontanals: «Mal testimonio dará (de sí) su hijo si es cierto lo que dice la mayor parte de la gente que le desposeerá en vida» ${ }^{7}$; senza note di commento, ma è evidente che su hijo non può che riferirsi a D. Sancho;

2) Joseph Anglade (parafrasi): «Riquier prévoit avec ses sujets que ses enfants le dépouilleront de son vivant de sa couronne» ${ }^{8}$;

3) Martín de Riquer: «en mala hora verá a su hijo, si dice verdad la mayoría de la gente: que vivo será por el desposeído», in nota chiarisce che si tratta dell' «infante don Sancho, el futuro Sancho IV el Bravo de Castilla»'

4) Carlos Alvar riprende la traduzione di Riquer e ossserva: «tras renunciar, a instancias de Gregorio X, al Imperio [Alfonso $\mathrm{X}]$ tuvo que relegar a segundo plano la teoría de Las Partidas, reconociendo a su hijo Sancho como heredero. A este momento

Ariel, 1975, III, p. 1621.

${ }^{5}$ Inequivocabile la strofe esordiale e, in modo particolare, il $s a$ di v. 5: «Qui $\cdot \mathrm{m}$ disses, non a dos ans, | que 1 laus me fos desgrazitz | del rey n'Anfos, de pretz guitz, | mot me fora greus afans; $\mid$ qu'er es tant vil tengutz $s a \mid$ e blasmatz ...».

${ }^{6}$ Avverto, per completezza di informazione, che mi è rimasto inaccessibile il contributo di René Nelli e Louis Alibert, «Actualité des troubadours: les troubadours de l'Aude», Pyrénées (Toulouse), 2 (1941), pp. 116-210 (che a p. 197 pubblicano anche il vers in esame). Dai contributi di chi ha potuto consultarlo (Riquer, che ne riprende il testo, e Alvar; cfr. infra) non sembrano emergere divergenze sostanziali quanto all'interpretazione del testo.

${ }^{7}$ Manuel Milà y Fontanals, De los trovadores en España, Barcelona, Libreria de Joaquin Verdaguer, 1861, p. 224. Lo studioso catalano, però, utilizzava l'edizione Pfaff (la sola disponibile all'epoca), che per il v. 19 legge Mal aueyra sos enfans ecc. (soluzione da rigettare; cfr. Monica Longobardi, «I vers», art. cit., p. 79).

${ }^{8}$ Joseph Anglade, Le troubadour Guiraut Riquier, op. cit., p. 157.

${ }^{9}$ Martín de Riquer, Los trovadores, op. cit., III, num. 346, p. 1622, traduzione e nota al v. 19. 
hace alusión el sirventés de Guiraut Riquier: los súbditos no podían estar contentos con su monarca que los había gravado con impuestos especiales para sostener los gastos de las pretensiones imperiales; al final, todos los esfuerzos fueron vanos y, a la vez que perdía el dinero, olvidaba la guerra de los benimerines ... Por otra parte, también se refleja en el sirventés el problema sucesorio» ${ }^{10}$;

5) Monica Longobardi: «guai se vedrà i suoi figli, se è vero quello che dice la maggior parte della gente, spodestarlo ancora vivente!»; in nota spiega che «la allusione è diretta alla usurpazione dei diritti di successione spettanti ai figli del primogenito di Alfonso, Fernando de la Cerda, (morto nell'agosto del 1275) ad opera del figlio minore di Alfonso, Don Sancho» ${ }^{11}$.

GrRiq, dunque, dando voce lirica a una non meglio specificata vox populi, metterebbe in guardia Alfonso $\mathrm{X}$ dal pericolo d'essere spodestato dai figli, in modo particolare dal suo secondogenito che, probabilmente non pago d'essere stato designato come erede della corona, starebbe tramando per impossessarsene anzitempo.

Una contestualizzazione più precisa di Qui $m$ disses nell'epoca (il 1276) e nell'ambiente (la corte castigliano-leonese) in cui fu composto potrebbe giovare alla comprensione del testo.

Prima di procedere, una precisazione metodologica (a scanso d'equivoci). Intento di questo contributo è ricostruire, in maniera il più possibile rigorosa e documentata, il contesto storico-politico in cui fu composto il vers riquieriano, questione fin ora mai approfondita dalla critica, ma a mio avviso essenziale per la corretta interpretazione di Qui.m.disses. Non è mia intenzione, invece, ritornare sulle relazioni tra Alfonso X e i trovatori provenzali, questione che non è strettamente pertinente al problema più circostanziato (per certi versi specialistico) che intendo affrontare in queste pagine e che è stata oggetto negli ultimi cinquant'anni d'una nutrita bibliografia, il cui riepilogo però non sarebbe possibile in questa sede, se non a patto di semplificazioni e imprecisioni ${ }^{12}$. Mi astengo quindi sia dal produrre uno status quaestionis, sia da quell'ingombrante accumulo bibliografico così in voga nella produzione critica attuale (ma, il più

${ }^{10}$ Carlos Alvar, La poesía trovadoresca en España y Portugal, Madrid, CUPSA Editorial, 1977, p. 227; stessa interpretazione in Id., Textos trovadorescos sobre España y Portugal, Madrid, CUPSA Editorial, 1978, p. 169.

${ }^{11}$ Monica Longobardi, «I vers», art. cit., p. 79.

${ }^{12}$ Oltre agli interventi di Valeria Bertolucci Pizzorusso citati alla nota 1, si tengano presenti almeno gli altri contributi riquieriani della stessa studiosa raccolti nel volume Ead., Studi trobadorici, Pisa, Pacini, 2010 e i saggi alfonsini (con ampie aperture verso i trovatori provenzali) ripubblicati da Vicenç Beltran, in La corte de Babel. Lenguas poéticas y política en la España del siglo XIII, Madrid, Gredos, 2005 (gli uni e gli altri con ampia bibliografia pregressa). Chi si avvicini per la prima volta a GrRiq (e alle sue relazioni con il Rei Sabio) può fare riferimento alla densa bibliografia allestita da Gerardo Larghi, s.v. «Guiraut Riquier» nel Dizionario Biografico dei Trovatori, op. cit., pp. 294-296, senza dimenticare che il repertorio bibliografico più aggiornato rimane sempre la $B E d T$ di Stefano Asperti ( $<$ http://www.bedt.it/BEdT_04_25/index.aspx $>$ ). 
delle volte, tutt'altro che necessario) e mi limiterò ad indicare, per singole questioni, i contributi imprescindibili o più recenti ${ }^{13}$. Nel prosieguo dell'indagine, invece, si ascolterà più di frequente -e in maniera più dettagliata- la testimonianza di fonti storiografiche antiche e moderne. La preferenza accordata a questo materiale non implica, ovviamente, disinteresse nei confronti della ricerca filologica degli ultimi cinquant'anni, né tanto meno intende sminuirne o sottovalutarne l'importanza: si tratta di questioni diverse affrontate e risolte da punti di vista (e con strumenti) diversi. Sono convinto che compito dell'interprete non sia soltanto quello di analizzare i valori estetici e formali d'un testo, ma anche quello di collocare il testo nel tempo e nello spazio, e nel caso di Qui.m disses 1'apporto della ricerca storiografica può produrre risultati apprezzabili.

2. Gli anni tra il 1274 e il 1276 sono un periodo difficile per il regno di Alfonso X: i negoziati relativi al fecho del Imperio -questione che nel 1274 porta il Re ad allontanarsi dalla Penisola per incontrare il Papa a Beaucaire ${ }^{14}$ - sono falliti e, nel 1275, i Benimerines di Abu-Yusuf invadono il Regno. I primi tentativi d'arginare l'avanzata islamica ottengono risultati catastrofici: nel luglio o agosto dello stesso anno (le fonti divergono) ${ }^{15}$, mentre è di stanza a Ciudad (all'epoca Villa) Real in attesa di rinforzi per marciare sull'Andalusia, muore l'Infante D. Fernando de la Cerda (primogenito di Alfonso $\mathrm{X}$ ed erede designato al trono) ${ }^{16}$; poco dopo (il 7 di settembre), D. Nuño González de Lara ${ }^{17}$, potente vassallo del Re, perde la vita nella battaglia di Écija, in Andalusia.

\footnotetext{
${ }^{13}$ A tal riguardo, preciso che i contributi presi in considerazione (Milà y Fontanals, Anglade, Riquer, Alvar e Longobardi) sono da considerare, non come 'punti di riferimento' per l'elaborazione d'uno status quaestionis sui trovatori di Alfonso X, ma -come è buona norma in ogni ricerca, non solo filologica- come 'punto di partenza' dell'indagine in quanto, nella pur vasta bibliografia riquieriana, sono gli unici contributi che si soffermano sull'interpretazione puntuale di Qui $\cdot m$ disses.

${ }^{14} \mathrm{Cfr}$. Histoire générale du Languedoc, Toulouse, Privat, 1885, IX, pp. 47-48. Prima della partenza, Alfonso X nominò reggente del Regno l'Infante D. Fernando de la Cerda (cortes di Burgos, 1274); cfr. Manuel González Jiménez, Alfonso X el Sabio, Barcelona, Editorial Ariel, 2004, p. 275.

${ }^{15}$ Agosto per la Crónica de Alfonso X; il 25 luglio per gli Annales Toledanos; il 24 luglio per la Crónica di Jofré de Loaysa; cfr. rispettivamente: Manuel González Jiménez, Crónica de Alfonso X, Murcia, Real Academia “Alfonso X el Sabio", 1999, p. 184; Antonio C. Floriano, «Annales Toledanos, III», Cuadernos de Historia de España, 43-44 (1967), pp. 154-187 [173]; Jofré de Loaysa, Crónica de los reyes de Castilla: Fernando III, Alfonso X, Sancho IV y Fernando IV (1248-1305), edición y tradución de Antonio García Martínez, Murcia, Real Academia “Alfonso X el Sabio", 1982, pp. 17-18.

${ }^{16}$ La designazione fu ufficializzata nelle cortes di Vitoria del 1255; cfr. Manuel González Jiménez, Alfonso X, op. cit., p. 88.

${ }^{17}$ Cfr. Crónica de Alfonso X, ed. cit., p. 184 e Manuel González Jiménez, Alfonso X, op. cit., p. 300. Si tratta di Nuño González de Lara el Bueno, da non confondere con il figlio omonimo (cfr. infra nota 43); cfr. Luis de Salazar y Castro, Historia genealógica de la Casa de Lara, 3 vols., Madrid, Imprenta Real, Mateo de Llanos y Guzmán, 1697, III, pp. 79-85 e, il più recente, Antonio Sánchez de Mora, «Nuño González de Lara: “El más poderoso omne que sennor ouiese
} 
Il secondogenito del Re, l'Infante D. Sancho, convenuto a Burgos con gli esponenti più in vista della nobiltà castigliano-leonese, si proclama «fijo mayor heredero del rey don Alfonso» ${ }^{18}$ e organizza una fulminea controffensiva militare ${ }^{19}$ : prima riscatta Ciudad Real, poi rafforza le zone di frontiera meridionali (soprattutto Cordova e Écija) e, infine, raggiunge Siviglia, dove ordina d' «armar la flota» per impedire ai Benimerines di ricevere rinforzi dall'Africa ${ }^{20}$. Solo il ritorno di Alfonso X, sul finire del 1275, non permette all'Infante di chiudere la partita con i Mori: preso atto che ogni pericolo imminente è ormai scongiurato, il Rei Sabio si accontenta d'una tregua biennale con Abu-Yusuf; soluzione che provoca malcontento nell'aristocrazia, che giudica inefficace l'intervento del $\mathrm{Re}^{21}$.

D. Sancho, però, tenta di trarre profitto dalla popolarità raccolta sul campo di battaglia ed esige il riconoscimento dei propri diritti di successione. Il primo incontro tra padre e figlio ha luogo, negli ultimi mesi del 1275, a Camarena (nei pressi di Toledo): 1'Infante vi giunge scortato da un imponente seguito di nobili che reclamano a gran voce il riconoscimento di D. Sancho come erede della corona ${ }^{22}$. Nonostante la solennità della situazione, Alfonso $\mathrm{X}$ sospende il giudizio $\mathrm{e}$ prende tempo. Non si pronuncia al riguardo neppure l'anno seguente, quando le cortes di Burgos (aprile 1276) riconoscono come legittima la successione di D. Sancho ${ }^{23}$. Che si tratti soltanto del riconoscimento d'una parte del Regno (l'aristocrazia), privo però dell'avallo del $\mathrm{Re}$, lo conferma la formula con cui gli Annales toledanos registrano l'avvenimento: «Anno Domini $\mathrm{M}^{\circ} \mathrm{CC}^{\circ} \mathrm{LXXVI}^{\circ}$. Magnates Regni castelle et Legione et Gallecie atque Lusitanie fecerunt omagium domino Sancio filio illustris Alfonssi Castelle regis et fratris dominis Fernandis» ${ }^{24}$; e sulla stessa linea si colloca la Crónica di Jofré de Loaysa, che ricorda come in quell'occasione anche i fratelli del Re (D. Manuel e D. Fradique) patrocinano la causa dell'Infante e

e más honrado de Espanna"», Historia, instituciones, documentos, 31 (2004), pp. 631-644.

${ }^{18}$ Crónica de Alfonso X, ed. cit., p. 186.

${ }^{19}$ Nelle operazioni belliche morì anche D. Sancho d'Aragona, Arcivescovo di Toledo (figlio di Jaime I e quindi cognato di Alfonso X), caduto a Martos il 20 ottobre del 1275 (cfr. Crónica de Alfonso X, ed. cit., p. 185). Antonio Ballesteros Beretta, Alfonso X el Sabio, Barcelona, El Albir, $1984^{2}\left(1^{\mathrm{a}}\right.$ ed. 1963), p. 184, misinterpretando la successione degli eventi fornita dalla Crónica (e non prestando attenzione agli Annales Toledanos; cfr. ed. cit., p. 173), ipotizzò che la morte dell'Infante D. Fernando dovette avvenire dopo quella dell'Arcivescovo D. Sancho, sul finire di ottobre o nei primi giorni di novembre del 1275.

${ }^{20}$ Crónica de Alfonso X, ed. cit., p. 187.

${ }^{21}$ Cfr. Joseph F. O'Callaghan, The Learned King: The Reign of Alfonso $X$ of Castile, Philadelphia, University of Pennsylvania Press, 1993, p. 238. Abu-Yusuf si ritirò in Marocco già nel dicembre del 1276, ma manteneva il controllo di numerosi avamposti sulla Penisola ed era evidente a tutti che le ostilità sarebbero presto riprese.

${ }^{22}$ Cfr. Crónica de Alfonso X, ed. cit., pp. 188-189 e Georges Daumet, Mémoire sur les relations de la France et de la Castille de 1255 à 1320, Paris, Fontemoing, 1913, pp. $22-23$ (datato e a volte impreciso, ma ancora utile, soprattutto per la raccolta dei documenti che pubblica).

${ }^{23}$ Cfr. Joseph F. O'Callaghan, The Learned King, op. cit., pp. 238-239.

${ }^{24}$ Annales Toledanos, ed. cit., p. 173. 
aggiunge che essi «eidem [a D. Sancho] tamquam futuro regi homagium prestiterunt $\rangle^{25}$.

La situazione di Alfonso X però è molto delicata: non è riuscito ad ottenere la corona imperiale -motivo per cui era assente dal Regno nel momento in cui Abu-Yusuf aveva sferrato l'attacco-, non è più giovane e non è nemmeno in perfetta salute. Durante il suo soggiorno a Vitoria (tra l'agosto del 1276 e il marzo dell'anno seguente) è tormentato da gravi problemi di salute, che fanno temere per la sua vita: è l'episodio che dà origine alla Cantiga CCIX de Santa Maria ${ }^{26}$, ma è anche l'episodio cui velatamente allude lo stesso D. Sancho, compromettendosi a terminare la costruzione (intrapresa dal padre) del monastero di Caleruega, «si alguna cosa aviniere a vos, lo que Dios no quiera, ante que el monesterio que vos facedes ...fuese acabado» ${ }^{27}$. Dati questi presupposti, è comprensibile che per larghissima parte della nobiltà, la scelta tra $\mathrm{D}$. Sancho -un giovane di 17 anni che aveva difeso con vigore il Regno in un momento d'estremo pericolo e continuava a partecipare attivamente alle questioni di governo (cfr. infra § 3)-e D. Alfonso de la Cerda - un bimbo di sei anni, la cui minore età, ad un'eventuale morte improvvisa del Re, avrebbe richiesto un periodo di reggenza e attratto, quindi, il Regno nell'orbita france$\mathrm{se}^{28}$ - si risolve a favore del primo (e rilevante è il fatto che persino $\mathrm{i}$ fratelli di Alfonso X appoggiano la successione di D. Sancho).

La legge del Regno (le Siete Partidas) gioca però a sfavore di tale designazione. In caso di morte prematura dell'Infante primogenito, la corona passa al primogenito dell'Infante morto:

Et esto usaron siempre en todas las tierras del mundo do el señorio hobieron por linage, et mayormente en España: ca por escusar muchos males que acaescieron et podrien aun seer fechos, posieron que el señorio del regno heredasen siempre aquellos que veniesen por liña derecha, et por ende establescieron que si fijo varón hi non hobiese, la fija mayor heredase el regno, et aun mandaron que si el fijo mayor moriese ante que heredase, si dexase fijo ó fija que hobiese de su muger legítima, que aquel ó aquella lo hobiese, et non otro ninguno ${ }^{29}$.

${ }^{25}$ Jorfré de Loaysa, Crónica, ed. cit., pp. 19-21.

${ }^{26} \mathrm{Cfr}$. Walter Mettmann (ed.), Alfonso X o Sábio, Cantigas de Santa Maria, 4 vols., Coimbra, Universidade de Coimbra (Acta Universatis Conimbrigensis), 1959-1972, cantiga CCIX: «Como el Rey Don Affonso de Castela adoeçeu en Bitoria e ouv'ha door tan grande que coidaron que morresse ende, e poseron-lle de suso o livro das Cantigas de Santa Maria, e foi guarido».

${ }^{27}$ Documento redatto a Burgos, l'8 novembre del 1277; cfr. Juan Loperráez Corvalán, Descripción histórica del Obispado de Osma, 3 vols., Madrid, Imprenta Real, 1788, III, num. LXXVI e Eduardo Martínez, Colección diplomática del Real Convento de Sto. Domingo de Caleruega, Vergara, El Santísimo Rosario, 1931, num. 28.

${ }^{28} \mathrm{D}$. Alfonso de la Cerda era figlio di Bianca di Francia, sorella di Filippo III l'Ardito.

${ }^{29}$ Las Siete Partidas del Rey Don Alfonso el Sabio, 3 vols., Madrid, Real Academia de la Historia, Imprenta Real, 1807, II, p. 132 (Partida II, título XV, ley II: Cómo el fijo mayor del rey ha adelantamiento et mayoría sobre los otros sus hermanos). 
La designazione di D. Sancho rappresenterebbe non solo l'abiura delle Partidas, la legge che lo stesso Re ha promosso e promulgato (e quindi, un atto iniquo nei confronti dell'Infante de la Cerda), ma anche e soprattutto un'intollerabile ingerenza della nobiltà; ingerenza quanto mai spiacevole proprio perché, in quel momento, Alfonso X sente minacciato il proprio prestigio personale. Il Re sa bene, inoltre, che qualunque decisione prenda, le conseguenze della scelta saranno gravi $^{30}$ : se pretende il rispetto delle Partidas, si attirerà contro l'ira dei nobili che, in larga parte, appoggiano D. Sancho e, anzi, l'ambizione del figlio potrebbe costargli una guerra civile (la Storia ha poi dimostrato che tali timori non erano infondati); se revoca i diritti di successione dell'Infante de la Cerda, scatenerà l'ira di Filippo l'Ardito (cognato di D. Fernando).

A tal riguardo, González Jiménez fa riferimento a non meglio specificati «compromisos adquiridos con Francia en las capitulaciones matrimoniales [tra Bianca di Francia e D. Fernando de la Cerda] de $1269 \rtimes^{31}$. Effettivamente, sia la Chronique di Guillaume de Nangis, sia la Crònica di Bernat Desclot menzionano un accordo, stipulato tra Luigi IX e Alfonso X, che ha per oggetto la successione al trono dei figli di Bianca e di D. Fernando. Il francese lo presenta come una clausola del contratto nuziale:

Blancha, filia regis Franciae Ludovici, datur a patre uxor Ferrando, primogenito regis Castellae, eo pacto quod primogenitus puerorum de ipsa procreandorum, nullius fraternitatis successione praejudicium inferente, avo vel patre ipsorum defunctis, regnum Hispaniae pacifice possideret ${ }^{32}$.

Il catalano, senza specificare le condizioni in cui fu stipulato, lo lascia cadere come inciso nel resoconto degli eventi occorsi dopo il rientro del Re nella Penisola:

${ }^{30}$ Cfr. Robert A. MacDonald, «Alfonso the Learned and Succession: A Father's Dilemma», Speculum, 40 (1965), pp. 647-653 [651-652]; Jerry Craddock, «Dynasty in Dispute: Alfonso X el Sabio and the Succession to the Throne of Castile and León in History and Legend», Viator, 17 (1986), pp. 197-219; Evelyn S. Procter, Curias y cortes de Castilla y León, Madrid, Editorial Cátedra, 1988, pp. 154-155; Joseph F. O’Callaghan, Las cortes de Castilla y León (1188-1350), Valladolid, Ámbito Ediciones, 1989, p. 39 e Id., The Learned King, op. cit., pp. 46-47 e 238-239.

${ }^{31}$ Manuel González Jiménez, «Sancho IV, Infante», Historia, instituciones, documentos, 28 (2001), pp. 151-216 [154]. Il primo compromesso nuziale fu firmato a Parigi il 28 settembre del 1266; Bianca ha 13 anni; D. Fernando appena 10 (e per questo motivo il contratto è stipulato in suo nome da due ambasciatori di Alfonso X); cfr. Gaspar Ibáñez de Segovia Peralta y Mendoza (Marqués de Mondejar), Memorias históricas del Rei D. Alonso el Sabio i observaciones a su chronica, Madrid, Joachin Ibarra editor, 1777, p. 524. Il matrimonio fu celebrato a Burgos, il 30 novembre 1269.

${ }^{32}$ Hercule Géraud (ed.), Chronique latine de Guillaume de Nangis, 2 vols., Paris, Jules Renouard et $\mathrm{C}^{\mathrm{ie}}, 1843$, I, pp. 235; corsivo mio. 
[Alfonso X] feu jurar a totes les gents del regne de Castella e de tota la terra son fill En Sancho per rey, e no volch que negun dels fills d'En Ferrando son fill que fos rey. Si quel rey Felip de França, de qui eren nebots, fills de sa sor, qui havia convinença ab lo rey de Castella que, apres de la mort d'En Ferrando fossen e deguessen esser sos nebots reys, fon molt yrat ${ }^{33}$.

L'ipotesi è suggestiva, ma manca dei necessari riscontri ${ }^{34}$. La covinença di cui parla Bernat Desclot non sembra aver lasciato tracce pergamenacee. La testimonianza di Guillaume de Nangis è in flagrante contraddizione con i documenti relativi al matrimonio di Bianca di Francia e D. Fernando de la Cerda ${ }^{35}$, in modo particolare con un atto di S. Luigi che ratifica i termini discussi con gli ambasciatori di Alfonso X (copia databile al settembre del 1266; cfr. infra). Il contratto, dopo il riepilogo dettagliato degli aspetti economici della questione (dote e vitalizio della sposa) si conclude con una clausola che regola l'eventuale vedovanza di Bianca: «si forte contingeret quod dicta filia nostra Blancha superviveret dicto Fernando, liceret ei libere, si vellet, redire in Franciam et haberet integraliter id quod nos in maritagium eidem dedimus et dotalicium suum superius dictum $\rangle^{36}$. È quanto meno sospetto però -in un'epoca in cui i matrimoni ad altro non servivano che a procacciare doti e rampolli- che, nel momento in cui si regola il futuro della coppia (fin anche a postulare le sorti di Bianca nel caso in cui il marito morisse prima di lei), non una sola parola sia dedicata al futuro dei figli che sarebbero nati da quell'unione; e tale silenzio non può che essere spiegato dal fatto che la successione al trono dei figli di Bianca e Fernando doveva essere garantita proprio dalla legge in vigore nel Regno di Castiglia-León. Per altro verso, che il patto menzionato dalle cronache possa essere scomparso in territorio iberico non è cosa che sorprenda (soprattutto se si considera che della corona si impadronisce D. Sancho), mentre stupisce che tale documento -d'importanza capitale in quanto avrebbe consentito ai Capetingi di avanzare diritti sulla corona di Castiglia-León- sia scomparso anche

\footnotetext{
${ }^{33}$ Bernat Desclot, Crònica, ed. de Miquel Coll i Alentorn, 2 vols., Barcelona, La Caixa, $1987^{3}$ [1949], cap. LXVI; corsivo mio.

${ }^{34}$ Hercule Géraud (ed.), Chronique latine, ed. cit., p. 235, nota 2 segnala che alcuni manoscritti della Chronique di Guillaume de Nangis mettono in relazione questo patto con un precedente accordo secondo il quale «rex sanctus Francice Ludovicus debebat in regno Hispanice ratione matris suae jus habere legitimum» (la madre di S. Lugi era Bianca di Castiglia, figlia di Alfonso VIII). L'editore commenta (ibidem): «ce droit d'hérédité eût été bien peu de chose, puisque Blanche de Castille avait trois sœurs dont elle n'était même pas l'ainée. Mais saint Louis pouvait faire valoir un acte d'Alphonse le Noble, son aïeul maternel, dont les seigneurs castillans avaient jadis réclamé l'exécution. Alphonse avait ordonné que si son fils unique D. Henri décédait sans postérité, (ce qui arriva en effet le 6 juin 1217), le royaume de Castille passerait à Louis, fils ainé de Blanche de Castille et de Louis fils de Philippe-Auguste».

${ }^{35}$ Gli atti relativi al matrimonio di Bianca e D. Fernando sono stati conservati tutti da fondi documentari francesi e si leggono, con essenziali note di commento, in Georges Daumet, Mémoire, op. cit., pp. 149-156 (Pièces justificatives III-VI).

${ }^{36}$ Ibidem, p. 153 (documento IV).
} 
in Francia, i cui fondi documentari hanno invece conservato il contratto nuziale. È vero che, come segnala Daumet, il contratto ratificato da Luigi IX è una «copie $d u$ temps sur un rouleau de parchemin, non datée» (ma databile al settembre del 1266) ${ }^{37}$, tuttavia, quale copista du temps, attivo presso la cancelleria capetingia, avrebbe omesso un simile dettaglio? La domanda retorica, ovviamente, non prova nulla, ma a quadrare il cerchio ci sono sia la preoccupazione dello stesso D. Fernando de la Cerda che, temendo l'ambizione e lo spirito di rivalsa del fratello ${ }^{38}$, affida in punto di morte i figli a D. Juan Núñez de Lara ${ }^{39}$, incaricandolo di vegliare sui diritti di successione del piccolo D. Alfonso ${ }^{40}$; sia la fretta e la solennità -ma, soprattutto, la fretta- con cui D. Sancho, sull'eco non ancora sopita dei pericoli corsi dal Regno, cerca di ottenere il riconoscimento paterno.

Come si è visto, però, Alfonso X tace. Tace sul finire del 1275 (a Toledo); tace nell'aprile del 1276 (a Burgos). Da entrambi i lati dei Pirenei, il silenzio del Re è per alcuni (colpevole) appoggio alle rivendicazioni di D. Sancho, per altri sostegno (altrettanto colpevole) all'Infante de la Cerda; e la situazione rischia di precipitare. La documentazione relativa al 1276 , sia di parte francese, sia di parte ispanica, non consente una ricostruzione completa degli eventi. '̀ plausibile pensare che Filippo l'Ardito -non importa in che modo (la sorella continuava comunque a vivere a corte) - sia venuto a conoscenza di quanto stava accadendo nella Penisola e abbia fatto pervenire ad Alfonso X le sue rimostranze ${ }^{41}$. Al 1276 risale una lettera alfonsina al Re di Francia -scritta evidentemente dopo l'adunanza burgalese- nella quale Alfonso X si dichiara pronto (e parla anche a nome dei nobili) a ritirare i «sacramenta et homagia facta eidem Sancio ${ }^{42}$. Ma tali garanzie non convincono né Filippo III, né quella parte della nobiltà (soprattutto i Lara) che fin dalla morte di D. Fernando avevano sostenuto la successione dell'Infante de la Cerda. Nel settembre del 1276, tre fidalgos di Alfonso X passano al servizio del Re di Francia: D. Juan Núñez de Lara (cui l'Infante D. Fernando

${ }^{37}$ Ibidem

38 'Qualità' di cui l'Infante D. Sancho aveva dato prova fin dai primi anni: emblematico il rifiuto di farsi armare cavaliere dal fratello D. Fernando; cfr. Crónica de Alfonso X, ed. cit., p. 50 e Ferran Soldevila (ed.), Crónica del rei Jaume I el Conqueridor, en Les Quatre Grans Cròniques, Barcelona, Selecta, 1971, cap. 495.

${ }^{39}$ Figlio e successore del Nuño González che muore a Écija. Luis de Salazar y Castro, Historia genealógica, op. cit., III, pp. 117 e ss. sbaglia nell'identificare le coordinate biografiche di D. Juán Núñez e indica come data di morte il 1276 (anno in cui abbandona la Penisola per mettersi al servizio di Filippo III l'Ardito; cfr. infra nota 44); cfr. Carlos Estepa Díez, «Dona Juana Núñez y el señorío de los Lara», E-Spania. Revue interdisciplinaire d'Études Hispaniques Médiévales, in rete, $<\mathrm{http} / / / \mathrm{e}-\mathrm{spania}$.revues.org/315> [16 aprile 2006].

${ }^{40}$ Crónica de D. Alfonso X, ed. cit., p. 184; cfr. Manuel González Jiménez, Alfonso X, op. cit., pp. 286-289.

${ }^{41}$ Cfr. Georges Daumet, Mémoire, op. cit., pp. 27-30.

${ }^{42}$ Cfr. Antonio Ballesteros Beretta, Alfonso X, op. cit., p. 790. 
aveva affidato i figli); D. Nuño González de $\operatorname{Lara}^{43}$ e D. Fernando Yáñez de Valverde ${ }^{44}$. La Chronica di Guillaume de Nangis inoltre riferisce che, in quello stesso mese, Filippo l'Ardito dichiara guerra ad Alfonso X e che l'esercito francese è già in marcia verso i Pirenei ${ }^{45}$.

Nel settembre del 1276, pertanto, tutto quello che si può affermare con ragionevole sicurezza, è che Alfonso X: 1) è ancora lontano dall'aver preso una decisione definitiva sulla successione al trono; 2 ) si trova a dover affrontare una possibile invasione francese.

Ma, mentre le truppe di Filippo III si avvicinano ai Pirenei (le rincontreremo fra poco), è tempo di tornare al vers di GrRiq.

3. Per i vv. 19-21,

Mala veyra sos efans, si.l pus de la gent ver ditz que vius n'er despostaditz!

l'interpretazione vulgata (cfr. § 1) presuppone che:

1) Alfonso $X$ sia schierato (o per lo meno connivente) con il figlio Sancho contro il nipote Alfonso de la Cerda;

2) la corte (o la maggior parte della corte) sia schierata con l'Infante de la Cerda contro il Re;

3) circolino rumori su una congiura di D. Sancho (vera o presunta, in questo momento poco importa) per estromettere il padre dal trono.

Tale lettura, però, è in aperta contraddizione con il contesto storicopolitico cui allude Qui $\cdot m$ disses, in quanto:

${ }^{43}$ Figlio di D. Juan Núñez e nipote omonimo del Nuño González morto a Écija nel settembre del 1276. Sui Lara, potente famiglia biscaglina più volte ribelle ad Alfonso X, si veda oltre a Luis de Salazar y Castro, Historia genealógica, op. cit. anche Estanislao Jaime de Labayru y Goicoechea, Historia general del senorio de Vizcaya, Bilbao - Madrid 1897 [ripubblicato da Ábaco Libros, Madrid, 1969] (datato, ma ancora utile soprattutto per la grande quantità di documenti raccolti e esaminati). Per le ribellioni dei Lara durante il regno di Alfonso X cfr. Antonio Ballesteros Beretta, Alfonso X, op. cit., 120-128; Vicenç Beltran, «Tipos y temas trovadorescos. I. Xemeno de Aybar», Zeitschrift für Romanische Philologie, CIV (1988), pp. 46-60; Id., «Tipos y temas trovadorescos. VI. García López de Alfaro y el ciclo de las hostilidades del Norte», Estudios Románicos, IV (1987-1989) [= Homenaje al profesor Luis Rubio], pp. 143-148; Id., «Tipos y temas trobadorescos IV. Pero da Ponte y la rebelión de don Lope Díaz de Haro», in Estudos portugueses. Homenagem a Luciana Stegagno Picchio, Lisboa, Difel, 1991, pp. 15-36; Fabio Barberini, «Un'infante ribelle, un prestito non restituito e il fecho del Imperio (Raimon de Tors, Per l'avinen pascor, BdT 410,6)», Studi Medievali (in stampa).

${ }^{44}$ Gli accordi stipulati dai Lara si leggono in Georges Daumet, Mémoire, op. cit., pp. 157 162 (documenti VII e VIII) e cfr. anche pp. 31-32. L'accordo stipulato da Fernando Yañez de Valverde si legge in Francisque Michel (ed.), Histoire de la guerre de Navarre en 1276 et 1277 , par Guillaume Anelier de Toulouse, Paris, Imprimerie Impériale, 1856, p. 624 e nota 1.

${ }^{45}$ Cfr. Georges Daumet, Mémoire, op. cit., pp. 40-43. 
1) Alfonso $X$ non è ufficialmente schierato né con il figlio, né con il nipote;

2) la corte (o la maggior parte della corte) è schierata con l'Infante D. Sancho;

3) D. Sancho non sembra avere, almeno nel settembre del 1276, alcun motivo per brigare contro il padre.

Nel vers di GrRiq, la «maggior parte della gente» del v. 20 non può che essere identificata con la corte stessa, ma attribuire all' entourage del Re sentimenti d'ostilità nei confronti di D. Sancho porterebbe ad una situazione paradossale: quella stessa corte che in ben due recenti occasioni -a Toledo, sul finire del 1275 e a Burgos, nell'aprile del 1276- ha sostenuto la designazione di D. Sancho come erede della corona, teme ora, trascorsi neppure 5 mesi dalle cortes di Burgos, un 'colpo di stato' da parte del candidato cui ha reso ufficialmente omaggio (e non si dimentichi che anche i fratelli del Re, D. Manuel e D. Fradique, appoggiano le ragioni di D. Sancho).

Altrettanto improbabile risulta la possibilità che D. Sancho intenda spodestare il Padre. Sebbene la Crónica de Alfonso $X$ riferisca che già all'indomani della morte del fratello, l'Infante si proclama «fijo mayor heredero del rey don Alfonso ${ }^{46}{ }^{4}$, la documentazione conservata non dà riscontro alle affermazioni del cronista: nei pochi diplomi siglati dall'Infante nel 1276 la scrizione non va mai al di là del titolo di cui D. Sancho era legittimamente detentore, «fijo mayor del muy noble [rey] don Alfonso, mío padre ${ }^{47}$; il titolo di erede, invece, appare per la prima volta in un documento del novembre del 1276 nel quale D. Sancho si firma come «fijo mayor y heredero» ${ }^{48}$ del Re, e questa formula comincia ad essere impiegata sistematicamente solo a partire dal $1277^{49}$, ovvero in epoca successiva alla composizione di Qui $\cdot$ m disses. L'itinerario di D. Sancho nell'anno 1276 non può essere ricostruito per intero; i documenti disponibili permettono solo di collocarlo a Valladolid in marzo, a Sabugal (zona di Riba Coa) in settembre, a Puebla de Valdeorras e Astorga tra novembre e dicembre ${ }^{50}$. Non è da escludere, però, che l'Infante -per seguire da vicino la questione relativa alla successione- accompagni Alfonso $X$ in tutti gli spostamenti compiuti in quell'anno: Toledo (gennaio-febbraio); Ávila (12

\footnotetext{
${ }^{46}$ Crónica de Alfonso X, ed. cit., p. 186.

${ }^{47}$ Documento del 14 novembre 1276; cfr. Luis Sánchez Belda, Documentos Reales de la Edad Media referentes a Galicia, Madrid, Dirección General de Archivos y Bibliotecas, 1953, num. 801.

${ }^{48}$ Documento del 28 novembre 1276; cfr. José Manuel Nieto Soria, «Algunas consideraciones sobre el patronato castillano-leonés en los siglos XIII e XIV», Anuario de Estudios Medievales, 15 (1985), pp. 203-228 [216].

${ }^{49}$ Atto relativo al Convento dei predicatori di Benavente (Vitoria, 3 marzo 1277); cfr. Antonio Ballesteros Beretta, Alfonso X, op. cit., p. 824.

${ }^{50} \mathrm{Cfr}$. Manuel González Jiménez, «Sancho IV», art. cit., p. 177 e Antonio Ballesteros Beretta, Alfonso X, op. cit., p. 824.
} 
febbraio); Arévalo (16-18 febbraio); Valladolid (25 febbraio-20 marzo); Vitoria (agosto 1276-marzo 1277) ${ }^{51}$. È quasi sicuro inoltre che D. Sancho sia a Burgos, nell'aprile del 1276, per assistere alle cortes, che riconoscono legittima la sua successione al trono ${ }^{52}$. La documentazione sottoscritta dall'Infante nel 1276 è particolarmente rilevante:

1) Valdeorras (senza indicazione del giorno e del mese): protezione accordata al Monastero di Melón ${ }^{53}$;

2) Valladolid, 6 marzo: conferma, in nome di Alfonso $X$, d'un privilegio concesso da Fernando III al concejo di Cadahalso de los Vidrios per la vendita di legname al mercato di Escalona ${ }^{54}$;

3) Jaén, 10 giugno: conferma, in nome di Alfonso X, dell'esenzione del portazgo ai ballesteros di Brihuega (privilegio non valido per Toledo, Siviglia e Murcia) ${ }^{55}$;

4) Sabugal, 1 settembre: carta de población $n^{56}$;

5) Valdeorras, 14 novembre: conferma della protezione accordata al Monastero di Melón e conferma d'un privilegio di Alfonso $\mathrm{X}^{57}$;

6) Astorga, 28 novembre: conferma d'un privilegio di Alfonso IX al vescovato di Astorga ${ }^{58}$

7) Astorga, 8 dicembre: privilegio concesso alla Chiesa di Astorga ${ }^{59}$.

E di analogo tenore i documenti firmati (o confermati) per tutto il $1277^{60}$. Se ne deduce quindi una partecipazione attiva di D. Sancho all'amministrazione del Regno che non lascia supporre tensioni gravi con il padre, o per lo meno non di gravità tale da giustificare rumori circa una possibile deposizione di Alfonso X da parte del figlio.

Si potrebbe certo ipotizzare che i vv. 19-21 di Qui.m disses riflettano soltanto una diceria dei detrattori di D. Sancho, da identificare (non mi pare ci siamo molte alternative) con i sostenitori dell'Infante de la Cerda. Questa ipotesi, però, mi sembra poco plausibile: il punto di riferimento di GrRiq è la corte alfonsina e, nel settembre del 1276, i difensori del piccolo Alfonso de la Cerda (i Lara e l'aristocrazia minuta ad essi legata) avevano abbandonato la Penisola per militare nelle

${ }^{51}$ Cfr. Manuel González Jiménez, «Sancho IV», art. cit., pp. 153-154.

${ }^{52}$ Cfr. Joseph F. O'Callaghan, The Learned King, op. cit., pp. 238-239.

${ }^{53}$ Cfr. Luis Sánchez Belda, Documentos Reales, op. cit., num. 800.

${ }^{54} \mathrm{Cfr}$. Manuel González Jiménez, «Sancho IV», art. cit., p. 177.

${ }^{55} \mathrm{Cfr}$. Manuel González Jiménez y M. Antonia Carmona Ruiz, Documentación e Itinerarío de Alfonso X el Sábio, Sevilla, Servicio de Publicaciones de la Universidad de Sevilla, 2012, p. 610 .

${ }^{56} \mathrm{Cfr}$. Antonio Ballesteros Beretta, Alfonso X, op. cit., p. 824.

${ }^{57}$ Cfr. Luis Sánchez Belda, Documentos Reales, op. cit., num. 801.

${ }^{58} \mathrm{Cfr}$. José Manuel Nieto Soria, «Algunas consideraciones», art. cit., p. 216.

${ }^{59}$ Cfr. Antonio Ballesteros Beretta, Alfonso X, op. cit., p. 824.

${ }^{60} \mathrm{Si}$ veda il regesto allestito da Manuel González Jiménez, «Sancho IV», art. cit., pp. 177-178. 
file francesi ${ }^{61} \mathrm{e}$, vista la severa reazione del $\mathrm{Re}^{62}$, è poco verosimile che altri rimasti a corte (ma non certo «la maggior parte della gente») osino perorare apertamente le ragioni dei figli di D. Fernando ${ }^{63}$.

Come conciliare allora i dati (la coerenza testuale) del vers di GrRiq con i dati (la coerenza contestuale) della Storia? Una prima inevitabile conclusione è che se GrRiq parla dall'interno della corte e riflette, quindi, il punto di vista della corte, la sua critica non può che avere per oggetto l'indugio di Alfonso X nel ratificare (dopo le cortes di Burgos dell'aprile del 1276) la successione di D. Sancho. GrRiq, quindi, si allineerebbe con i partigiani del secondogenito del Re. Conclusione apparentemente ardita, ma coerente con il contesto storico cui allude il vers.

Le prime tre coblas di Qui.m disses costruiscono un crescendo, nel quale il discredito caduto su Alfonso X (indefinito nella cobla I) si va progressivamente precisando in ordine alle accuse e agli accusatori. La cobla II menziona «moutz homes ... | que li foran valedor» (vv. 10-11) se solo il Re fosse risoluto nella guerra come lo è nel donare («si guerra l'agues sabor | tant quon a cor de donar», vv. 1213). Il referente più immediato di questi versi è ovviamente la tregua stipulata l'anno precedente con i Benimerines, proprio nel momento in cui D. Sancho li avrebbe debellati senza grandi difficoltà ${ }^{64}$, ma questo particolare aspetto del carattere di Alfonso X può essere utile anche per comprendere la cobla III, nella quale ai «moutz homes» della II corrisponde ora «la maggior parte della gente» e il motivo di biasimo è legato al pleito successorio.

Ai vv. 19-21, editori e traduttori concordano nell'attribuire a vius e $n e$ (v. 21: «que vius $n$ 'er despostaditz!») valore, rispettivamente, di complemento predicativo del soggetto («vivo» o «da vivo») e di complemento d'agente («da loro» scil. «dai suoi figli»). Che nel settembre del 1276 Alfonso X possa essere spodestato dai suoi figli è altamente improbabile, ma c'è un altro elemento che, a mio avviso, suscita perplessità: perché il Re dovrebbe essere vius despostaditz, «spodestato da vivo»? È forse possibile spodestare un Re 'da morto'? Se vius è un complemento predicativo del soggetto, da riferire quindi ad Alfonso X, che complemento di informazione apporta al messaggio del trovatore che non sia quello già insito nel verbo, ovvero, che

${ }^{61} \mathrm{Cfr}$. supra e nota 44. Secondo i dati che si ricavano dai documenti pubblicati da Daumet e da Michel, con i Lara abbandonarono il servizio di Alfonso X ben 416 vassalli tra fidalgos, ricoshombres e caballeros.

${ }^{62}$ Ai Lara furono confiscati titoli e terre cfr. più avanti nota 69.

${ }^{63}$ Tra i sostenitori dell'Infante de la Cerda la testimonianza di Guillume de Nangis, la Crónica de Alfonso $X$ e quella di Bernat Deslot annoverano anche D.na Violante, ma la fuga rocambolesca della Regina, che nel 1278 riparò in Aragona insieme alla nuora e ai nipoti, «no tiene nada que ver con el pleito sucesorio sino más bien con el empeoramiento de las relaciones de la real pareja, dificultadas por los violentos cambios de humor del rey» (Manuel González Jiménez, «Sancho IV», art. cit., p. 155 e cfr. nota 22).

${ }^{64}$ Cfr. supra nota 21. 
per 'spodestare' un Re è necessario che egli sia in carica e, quindi, 'vivo'? Credo che, senza alcun intervento sulla lezione del testo, sia possibile attribuire: a vius valore avverbiale, «presto» ${ }^{65}$; a $n e$ valore di complemento di causa ${ }^{66}$, «a causa loro» scil. «a causa dei suoi figli». Tradurrei, pertanto, in questo modo: «a suo discapito vedrà i suoi figli, se è vero ciò che dice la maggior parte della gente, perché presto sarà spodestato a causa loro».

Nel settembre del 1276, l'esercito francese è già acquartierato sui Pirenei e la documentazione iberica non reca traccia dell'organizzazione d'una controffensiva anti-francese. I rapporti tra Alfonso X e Filippo III procedono, infatti, per via diplomatica, ma non sembrano produrre risultati apprezzabili. La lettera che pochi mesi prima il Rei Sabio aveva inviato al Re di Francia, dichiarando (anche a nome dell'aristocrazia) la disponibilità a rivedere la questione dinastica ${ }^{67}$, non è riuscita a dissuadere Filippo III dal marciare in armi contro il Regno di Castiglia-León e le trattative intavolate dopo l'arrivo dell'esercito sui Pirenei non sembrano promettere niente di buono: al 7 novembre del 1276 risale un accordo, stipulato a Vitoria da Alfonso $\mathrm{X}$ e Filippo III, secondo il quale il primo si impegna a ridiscutere il problema della successione in nuove cortes da convocare «postea de instanti festivitate Nativitatis Domini infra unum annum», il secondo «possit ad nostram congregatam curiam pro dicto negotio aliquos sapientes et viros providos transmittere, qui ... ostendant et pro eo advocent in curia supradicta $\rangle^{68} \mathrm{e}$ entrambi giurano di rimettersi senza ulteriori recriminazioni al verdetto di tale assise ${ }^{69}$.

Quest'accordo -che, com'è noto, rimase lettera morta- è successivo di appena due mesi alla composizione di Qui.m disses, ma decisioni di questo tipo non si prendono dalla notte al mattino ed è plausibile che, già nel settembre del 1276 , con l'esercito francese di stanza sul confine, le trattative diplomatiche di Alfonso X tenessero banco nelle conversazioni di corte. Se si considera, allora, che

${ }^{65} \mathrm{Cfr}$. Walther von Wartburg, Französisches Etymologisches Wörterbuch, Tübingen, Mohr Siebeck Verlag, 1948-1949 (rist. dei voll. I-II/i), Basel, R. G. Zbinden \& Co. Verlag, 1946-1950 (voll. II/ii-V), Basel, R. G. Zbinden \& Co. Verlag, 1969 ss. (vol. VI ss.), XIV, p. 583a (s.v. viou: «promptement») e cfr. anche le precisazioni di François Zufferey, «Apr. viu(s) "vite". À propos d'un passage de Na Castelloza», Cultura Neolatina, LXVIII (2008), pp. 311-318 [314].

${ }^{66}$ Valore non infrequente; cfr. Frede Jensen, Syntaxe de l'ancien occitan, Tübingen, Max Niemeyer Verlag, 1994, § 674.

${ }^{67}$ Cfr. Antonio Ballesteros Beretta, Alfonso X, op. cit., p. 790.

${ }^{68} \mathrm{Il}$ testo dell'accordo si legge in Francisque Michel (ed.), Histoire de la guerre de Navarre, op. cit., pp. 651-653 [le due cit. a p. 652].

${ }^{69}$ Contestualmente a questo accordo, i due sovrani siglano, lo stesso giorno (e lo confermano il dì seguente), anche un documento relativo ai Lara e ai nobili che li hanno seguiti al servizio del Re di Francia (cfr. supra nota 44) nel quale Alfonso si impegna a perdonarli e a restituire loro i possedimenti confiscati: «fuit ... concordatum quod, ad preces illustris regis Francorum, nos Johannem Nuniz et fratrem suum, et milites et nobiles et alios qui cum eis modo sunt, et familiam suam ad amorem nostrum et graciam admittamus, et quod demus eis tantum de avere nostro quantum tenere de nobis solebant, donec escadat nobis terra quom eis dare possimus») (testo in Francisque Michel, ed., Histoire de la guerre de Navarre, op. cit., p. 653). 
gli ultimi due anni di governo del Re non avevano brillato per iniziative contundenti - prima la precaria tregua biennale stipulata con Abu-Yusuf (dicembre 1275), poi il rifiuto di prendere posizione sulla designazione di D. Sancho come erede (aprile 1276)- è verosimile supporre che la nobiltà tema non solo che Alfonso X sia sul punto di sconfessare la decisione delle cortes di Burgos, ma anche e soprattutto che le trattative del Re non saranno in grado di scongiurare la guerra con la Francia, una guerra che sarebbe potuta costare il trono ad Alfonso X e l'indipendenza al regno di Castiglia-León.

Vius, in accezione avverbiale, esprimerebbe quindi il senso di imminente pericolo e di incertezza diffusa derivante proprio da questa situazione: pericolo dovuto alla vicinanza dell'esercito francese; incertezza dovuta all'esito delle trattative di Alfonso X con Filippo l'Ardito. GrRiq, tastando il polso ai malumori della corte (v. 20: «si·1 pus de la gent ver ditz»), ne raccoglie le preoccupazioni più urgenti e ammonisce il Re a (re)agire con maggiore risolutezza, altrimenti mala veyra sos enfans, «a suo discapito» (o forse anche «per sua vergogna») «vedrà i suoi figli» in quanto, presto, $n$ 'er despostaditz, «sarà spodestato a causa loro», ovvero: per non aver voluto difendere energicamente le ragioni dei suoi figli, soprattutto quelle di D. Sancho, che la «maggior parte della gente» aveva già riconosciuto come erede al trono.

4. La lettura proposta è più coerente con il contesto storico-politico e non sembra in disaccordo con il resto vers. Nella cobla IV GrRiq mette da parte le voci raccolte a corte per esprimere un ammonimento indirizzato al Re:
Ab dreg a volgut renhar ez ab pretz ez ab valor, creyssen de terr'ab lauzor le reys n'Anfos, que Dieus gar; ez aras deu mielhs e may voler dreg e patz dos tans, sol que non si'escarnitz, per que de Dieu si'aizitz, e sos pretz no's desenans.

Il passato (tempo verbale) che apre la cobla - a volgut- non implica necessariamente una frattura con il passato (tempo trascorso), ma traccia piuttosto una linea di continuità: il Re ha sempre governato secondo giustizia e ora più che mai deve (v. 32: «aras deu mielhs e may») garantire giustizia (dreg) e pace (patz).

Questi versi riflettono il dilemma che Alfonso X affronta tra il 1275 e il $1276^{70}$ e per comprendere appieno il vers di GrRiq è neces-

${ }^{70}$ Diversa interpretazione (ma, stranamente, senza argomentazione) è in Carlos Alvar, $L a$ 
sario chiedersi che tipo di dreg e patz invoca il trovatore. Ragionando in linea di principio, vale a dire secondo la legge di successione promulgata nelle Partidas, l'unica azione del Re che, al tempo stesso, assicurerebbe pace e giustizia è rompere gli indugi e riconoscere definitivamente il diritto di successione dell'Infante de la Cerda (questione che, per altro, tormenterà Alfonso X fino al 1281, scatenando la guerra civile che, negli ultimi quattro anni del suo regno, lo vide contrapposto al figlio $)^{71}$; ma, visto il favore di cui D. Sancho gode presso l'aristocrazia castigliano-leonese, è difficile pensare che questa soluzione avrebbe garantito pace nel Regno. Quanto rilevato a proposito delle coblas II-III, pertanto, porterebbe a concludere che la «pace» invocata da GrRiq nella cobla IV non sia quella da stipulare con il Re di Francia, ma quella da garantire a tutti i costi nel Regno di Castiglia-León, e l'unica azione che lo permetterebbe è, in accordo con la prima parte del componimento e con i dati del contesto storico, il riconoscimento ufficiale della successione di D. Sancho.

Tale riconoscimento, però, può essere definito 'giusto' e 'gradito a Dio'? Per la nobiltà, che nelle cortes di Burgos (aprile 1276), ha riconosciuto legittima la successione di D. Sancho, le Partidas non hanno costituito un grave ostacolo: in assenza di Alfonso X (impegnato a discutere con il Papa d'un titolo imperiale che mai gli sarà conferito) e di fronte al grave pericolo sofferto dal Regno, D. Sancho ha dimostrato di meritare la corona sul campo di battaglia, inoltre, ad un'eventuale improvvisa morte del Re (ipotesi non del tutto peregrina, vista la salute malferma di Alfonso X), il suo secondogenito potrebbe governare il Regno senza alcun periodo di reggenza (scongiurando così le ingerenze francesi che avrebbero invece accompagnato D. Alfonso de la Cerda) ${ }^{72}$. Si tratta d'una scelta pratica, quasi di Realpolitik, ma che non è totalmente in contrasto con l'ideologia soggiacente alla 'teoria regale' depositata nelle Partidas.

Nella Partida segunda, «que fabla de los emperadores, et de los reyes et de los otros grandes señores ${ }^{73}$, la monarchia è presentata come istituzione necessaria alla società: il Re è al contempo «corazón et alma del pueblo» e «cabeza del regno» ${ }^{74}$, ma soprattutto il Re è espressione della volontà di Dio, che lo ha scelto come suo

poesía trovadoresca, op. cit., p. 227 che ritiene che la cobla IV di Qui:m disses alluda al fecho del Imperio: «incluso las ideas imperialistas del rey castellano hallan su eco en esta composición».

${ }^{71} \mathrm{Cfr}$. più avanti nota 82 .

${ }^{72} \mathrm{~L}$ 'assenza d'un sovrano, come anche la presenza d'un sovrano inadeguato ai suoi compiti, mette in pericolo l'intero regno; cfr. Walter Ullmann, Principi di governo e politica nel Medioevo, Il Mulino, Bologna, 1972, p. 161.

${ }^{73}$ Las Siete Partidas, ed. cit., II, p. 3 (Prólogo).

${ }^{74}$ Ibidem, II, p. 7 (Partida II, título I, ley V). Su questa teoria 'organicistica' del potere regale -diffusasi in Europa grazie al Policraticus di Giovanni di Salisbury- fondamentale Ennst Hartwig Kantorowicz, I due corpi del re, Torino, Einaudi, 1989. Cfr. anche José Manuel Nieto Soria, Fundamentos ideológicos del poder real en Castilla (siglos XIII-XIV), Madrid, Eudema, 1988, p. 93 e Antony Black, El pensaniento político en Europa, 1250-1450, Cambridge, Cambridge University Press, 1996, pp. 20-36. 
delegato in terra ${ }^{75}$. Questi presupposti teologici interessano in modo particolare anche la successione regia: Dio sceglie il Re o facendolo nascere primogenito nella famiglia regale - «Dios lo escogió quando quiso que nasciese primeramente que los otros sus hermanos» ${ }^{76}-0$ aprendogli in altro modo la strada verso il trono.

Nel primo caso la Partida segunda concilia l'antichissima tradizione giudaica della sacralità del primogenito - «ca segunt él [Dio] dixo á Moysen en la vieja ley, todo másculo que nasciese primeramente serie llamado cosa santa de Dios» ${ }^{77}-$ con il più recente 'diritto di rappresentazione', di ascendenza romano-giustinianea ${ }^{78}$; per questo motivo «si el fijo mayor moriese ante que heredase, si dexase fijo ó fija que hobiese de su muger legítima, que aquel ó aquella lo hobiese, et non otro ninguno» ${ }^{79}$.

Nel secondo caso, le circostanze che permettono a un non primogenito di ascendere al trono sono tre:

1) quando lo gana por avenencia de todos los del regno que lo escogen por señor, non habiendo pariente que deba heredar el señorío del rey finado por derecho;

2) quando alguno casa con dueña que es heredera de regno, que maguer él non venga de linage de reyes, puédese llamar rey después que fuere casado con ella;

3) por otorgamiento del papa ó del emperador quando alguno dellos face reyes en aquellas tierras en que han derecho de lo facer ${ }^{80}$.

La volontà di Dio, quindi, esclude D. Sancho dalla successione sia nell'uno, sia nell'altro caso. Ma la volontà di Dio è un libro che richiede interpretazioni pazienti e flessibili, perché a volte, come recita l'adagio portoghese, Deus escreve direito por linhas tortas. In un passo della Crónica de Alfonso X, D. Sancho ricorda al padre che è stato Dio stesso ad aprirgli la strada verso il trono:

Sennor. Non me fezisteis vos, mas fízome Dios et fizo mucho por me fazer, ca mató a un mi hermano que era mayor que yo, e era vuestro heredero destos regnos, si el biuiera más que vos; e non lo mató por ál sy non porque lo heredasse yo después de vuestros días ${ }^{81}$.

${ }^{75}$ Las Siete Partidas, ed. cit., I, p. 2 (Prólogo generale).

${ }^{76}$ Ibidem, II, p. 131 (Partida II, titulo XV, ley I).

${ }^{77}$ Ibidem, II, p. 132 (Partida II, titulo XV, ley II).

${ }^{78}$ Istituto giuridico introdotto dalle Novellae Constitutiones di Giustiniano (cfr. novella 119); cfr. Antonio Pérez Martín, «Fuentes romanas en las Partidas», Revista de Historia del Derecho Español, 4 (1992), pp. 215-246 [242-244].

${ }^{79}$ Las Siete Partidas, ed. cit., II, p. 132 (Partida II, titulo XV, ley II).

${ }^{80}$ Ibidem, II, p. 10 (Partida II, titulo I, ley IX: En quántas maneras se gana el regno derechamente).

${ }^{81}$ Crónica de Alfonso X, ed. cit., p. 219. 
L'episodio riferito dalla Crónica ha luogo cinque anni dopo la composizione di Qui $\cdot m$ disses $^{82}$, e la Crónica stessa è di molto successiva al regno di Alfonso X, tuttavia non mi sembra inverosimile sostenere che lo stesso argomento, che D. Sancho presenta al padre nel resoconto di Fernán Sánchez de Valladolid ${ }^{83}$, fosse utilizzato già nel 1276 -ovviamente con minore acrimonia- anche dai sostenitori del secondogenito del Re. La monarchia castigliana, secondo la 'teoria' della Partida segunda, si configura (è una definizione di Nieto Soria) come un «feudalismo teológico» ${ }^{84}$ : il Re è vicario temporale di Dio in terra $\mathrm{e}$, in quanto tale, il Re deve rispettare e temere la volontà divina «como él quiere que lo teman los suyos» ${ }^{85}$. Dati questi presupposti, allora, è abbastanza plausibile supporre che, la morte di D. Fernando e l'esito vittorioso della controffensiva di D. Sancho contro i Benimerines fossero interpretati, da buona parte dei sostenitori del secondogenito del Re, come segni della volontà divina che additava quale erede al trono, non l'Infante de la Cerda, ma lo stesso D. Sancho. E non è da

${ }^{82}$ Si riferisce a un colloquio che D. Sancho e Alfonso X ebbero in occasione delle cortes di Siviglia del 1281. Sebbene D. Sancho fosse stato ufficialmente proclamato, con l'avallo del Re, erede della corona nelle cortes di Segovia (celebrate nell'agosto del 1278)-cfr. Miguel C. Vivanco Gómez, Documentación del Monasterio de Santo Domingos de Silos (1255-1300), Silos y Burgos, Abadía de Silos, 1995, num. 253-, le minacce del Re di Francia e le pressioni del Papa avevano indotto Alfonso $\mathrm{X}$ a riconsiderare la questione. Un primo tentativo di accordo con Filippo III ha luogo nel dicembre del 1280: i due sovrani si incontrano il 25 a San Sebastián e il 30 a Bayonne; Alfonso X non ha intenzione di revocare la successione di D. Sancho, ma offre al nipote il regno di Jaén e una rendita di 500 libre in terreni; ambedue le concessioni sono subordinate all'omaggio formale di Alfonso de la Cerda a D. Sancho (cfr. Antonio Ballesteros Beretta, Alfonso X, op. cit., pp. 918-919 e Crónica de Alfonso X, ed. cit., pp. 210-212). A questo episodio è dedicato il vers XIII di GrRiq, S'ieu ja trobat non agues (composto nel dicembre del 1280, quando il trovatore aveva già abbandonato la corte alfonsina). Il fallimento di questo accordo $\mathrm{e}$ la prosecuzione delle trattative per tutto il 1281 allarmano D. Sancho che, di fronte all'intenzione del padre di voler comunque venire a patti con Filippo III, rompe definitivamente i rapporti con il Re dando inizio a una guerra civile che prosegue fino al 1284 (morte di Alfonso X). Su origini e sviluppo del conflitto cfr. José Manuel Nieto Soria, Sancho IV (1284-1295), Palencia, Diputación Provincial de Palencia, Ediciones La Olmeda, 1994, p. 37 e ss.; Manuel González Jiménez, «Sancho IV», art. cit., pp. 167 e ss.; Id., Alfonso X, op. cit., pp. 343 e 348-349; Braulio Vázquez Campos, Los adelantados mayores de la frontera o Andalucia (siglos XIII-XIV), Sevilla, Diputación de Sevilla, Camas, 2006, pp. 181 e ss.

${ }^{83}$ Autore (Fernán Sánchez de Valladolid) e periodo di composizione (1344-1350) sono stati oggetto, tra la fine del s. XIX e la prima metà del successivo, d'un lungo e complesso dibattito che non ha però apportato argomenti di peso atti a revocare in dubbio questi dati; ottima sintesi in Casto María del Rivero, Índice de las personas, lugares y cosas notables que se mencionan en las tres Crónicas de los reyes de Castilla: Alfonso X, Sancho IV y Fernando IV, Madrid, CSIC, 1943, pp. 5-36, da integrare con Diego Catalán, Un cronista anónimo del siglo XIV (la «Gran Crónica de Alfonso XI». Hallazgo, estilo, reconstrucción), La Laguna, Universidad de La Laguna, 1955. Spunti interessanti in Michel Garcia, «L'historiographie et les groupes dominants en Castille. Le genre chronistique d'Alphonse X au chancelier Ayala», in Augustín Redondo (ed.), Les groupes dominants et leur(s) discours. (Domanine ibérique et latinoaméricain), Paris, Service de Publications Université de la Sorbonne Nouvelle-Paris III, 1984, pp. 53-70 [65-66].

${ }^{84}$ José Manuel Nieto Soria, Fundamentos, op. cit., p. 98.

${ }^{85}$ Las Siete Partidas, ed. cit., II, p. 16 (Partida II, titulo II, ley XIII). Allo speciale rapporto del Re con Dio è dedicato per intero il título II della Partida segunda: «quál debe el rey seer en conoscer, et amar et temer á Dios» (Ibidem, II, pp. 14-18). Il rispetto della volontà divina, inoltre, è un punto di riferimento costante per tutto il resto della Partida segunda. 
escludere, quindi, che la questione fosse presentata proprio in questi termini ad Alfonso X. Ad aggirare l'ostacolo della legge di successione interveniva, del resto, un altro articolo della Partida segunda che attribuisce al Re facoltà di modificare leggi e costumi se quelli esistenti possono rappresentare un pericolo per il Regno:

El poderío que el [il Re] ha es en dos maneras. La vna, de derecho. $E$ la otra de fecho. E aquel que ha segund derecho es este, que puede fazer ley e fuero nueuo, e mudar el antiguo, si entendiere, que es pro comunal de su gente. E otro si quando fuesse escuro, ha poder de lo esclarecer. E puede otrosi toller la costumbre vsada, quando entendiere que era dañosa, e fazer nueua que fuesse buena ${ }^{86}$.

E d'altra parte, il concetto di 'diritto' in epoca medievale è molto diverso dal nostro e bisognerebbe evitare di valutare la giurisprudenza antica con i parametri moderni ${ }^{87}$.

Nel 1276, GrRiq risiede a corte da più d'un decennio e gode ormai di grande familiarità con il Re e con il suo entourage. La Suplicatio sui giullari $(1264)^{88}$ è quasi contemporanea alla conclusione della prima stesura delle Partidas (redatte, secondo l'opinione più accreditata, tra il 23 giugno 1256 e il 28 agosto $1265^{89}$ ). I fondamenti teologici dell'attività legislativa di Alfonso X non dovevano quindi essere ignoti al trovatore e mi sembra plausibile suppore che proprio a tali fondamenti facciano allusione i vv. 32-36 di Qui $\cdot m$ disses:
ez aras deu mielhs e may
voler dreg e patz dos tans, sol que non si'escarnitz, per que de Dieu si'aizitz, e sos pretz no's desenans.

\footnotetext{
${ }^{86}$ Las Siete Partidas, ed. cit., II, p. 16 (Partida II, título I, ley II).

${ }^{87}$ Cfr. Manuel García Pelayo, «La idea medieval del derecho», Revista de la Facultad de Derecho, 23 (1962), pp. 9-65.

${ }^{88} \mathrm{Sul}$ questo importante testo, sulla responsiva Declaratio (pronunciata dal Re sapiente, ma composta dallo stesso GrRiq) e sul contesto culturale in cui fu composto il dittico si veda: Valeria Bertolucci Pizzorusso, «La supplica di Guiraut Riquier e la risposta di Alfonso X», Studi Mediolatini e Volgari, 14 (1966), pp. 9-136; Ead., «Per una rilettura della Supplica sui giullari di Guiraut Riquier», in Retórica medieval. ¿Continuidad o ruptura? (Actas del Simposio internacional de Granada, enero de 1995), Granada, Publicaciones de la Universidad de Granada, 1966, pp. 35-44 (oggi ripubblicato in Ead., Studi trobadorici, op. cit., pp. 105-111) e Ead., «Per una nuova edizione del libre di Guiraut Riquier», art. cit.

${ }^{89}$ Sulle Partidas (e i non pochi problemi che sollevano) si vedano: José Sánchez-Arcilla Bernal, «La obra legislativa de Alfonso X el Sabio. Historia de una polémica», in Ana Domínguez Rodríguez e Jesús Montoya Martínez (eds.), El scriptorium alfonsi de los libros de astrología a las «Cantigas de Santa María», Madrid, Editorial Complutense, 1999, pp. 17-81 e il più recente, José Manuel Nieto Soria, «Las Siete Pardidas de Alfonso X», in Feliciano Novoa Portela et alii (eds.), Historia de Europa a través de sus documentos, Barcelona, Lunwerg Editores, 2012, pp. 64-83; ambedue con ampia rassegna della bibliografia pregressa.
} 
GrRiq ammonisce il Re ad agire per il bene del Regno. Se la volontà divina vuole condurre D. Sancho al trono, Alfonso X non si opponga, solo in questo modo egli potrà:

1) assicurare dreg, ovvero, agire secondo giustizia, in quanto la designazione di $\mathrm{D}$. Sancho non si opporrebbe alla volontà di Dio e il Re ha facoltà -ricevuta direttamente da Dio- di modificare la legge per il bene del Regno (e in effetti, nel passaggio della Partida segunda citato poco sopra il cambio delle leggi è una delle maniere in cui il Re esercita il suo potere de derecho);

2) assicurare patz («pace», ma anche «concordia», «unità») all'interno del suo Regno, in quanto la maggior parte della nobiltà è ufficialmente schierata con D. Sancho;

3) evitare d'essere escarnitz dagli uomini (quindi dalla sua corte) e, soprattutto, riuscire 'gradito' a Dio.

Data l'indecisione del $\mathrm{Re}$-che rende molto complessa la situazione- e data, soprattutto, la posizione subalterna del trovatore, il 'consiglio' 90 è offerto in forme molto caute (vv. 37-40 e 43-45), il che, però, non impedisce a GrRiq di formulare un ultimo ammonimento (vv. 41-42):

pero aitant li diray

que reys deu amicx amar

che sembra suggerire al Re di ripassare la Partida segunda e la sua complessa serie di doveri regali; compito d'un re infatti è:

haber [malquerencia]... contra aquellos que facen al rey o al regno traición, ... que deben ser escarmentados en todos guisas sin ninguna merced; ca el rey contra los malos, en quanto en su maldat estodieren, siempre les debe haber mala voluntad, porque si desta guisa non lo feciese, non podrie facer justicia complidamiente, nin tener su tierra en paz nin mostrarse por bueno; mas debe haber buena voluntad á los buenos, et querer que vivan en paz: et faciendo asi acordará con las palabras que dixieron los ángeles por mandado de Dios á los pastores quando nació Iesu Cristo, que era fecha loor á Dios en los cielos, et dada en la tierra paz á los homes de buena voluntad. Onde el rey que de otra guisa hobiese malquerencia, si non como en esta ley dice por derecha razón, seria malquisto de Dios et de los homes ${ }^{91}$.

${ }^{90}$ Quello di 'consigliere' del Re sapiente è, in effetti, un ruolo cui GrRiq aspirerà (senza mai riuscire ad ottenerlo) durante l'intero soggiorno presso la corte castigliano-leonese. Su queste ambizioni frustrate si veda ora il recente intervento di Valeria Bertolucci Pizzorusso, «Conseil: un motivo/tema nella poesia dei trovatori», in Vicenç Beltran, Tomàs Martínez, Irene Capdevilla (eds.), 800 anys després de Muret. Els trobadors i les relacions catalanooccitanes, Barcelona, Edicions de la Universitat de Barcelona, 2015, pp. 75-100.

${ }^{91}$ Las Siete Partidas, ed. cit., II, p. 34 (Partida II, título V, ley XIII); corsivo mio. 
Si noti che i doveri del Re sanciti in questo comma (facer justicia complidamente; tener su tierra en paz; mostrarse por bueno e non essere malquisto de Dios et de los homes) sono gli stessi ai quali esorta GrRiq nella cobla IV del suo vers ( Ab dreg a volgut renhar ... | le reys n'Anfos ... | ez aras deu mielhs e may | voler dreg e patz dos tans, | sol que non si'escarnitz, | per que de Dieu si'aizitz»). E la corrispondenza non mi sembra casuale. Ancorché sia costretto a esprimersi con cautela, GrRiq spera che il Re avrà abbastanza discernimento per comprendere i suoi consigli. Se da un lato, l'esitazione di Alfonso X è dovuta (in parte) al rispetto della legge -quella che assegna la corona all'Infante de la Cerda-, dall'altro GrRiq rammenta al Re che la stessa legge impone al sovrano di rimettersi al consiglio de los buenos -nel caso di specie, quelli che, sostenendo D. Sancho, hanno permesso all'Infante di salvare il Regno dai Benimerines, e di rendere così un grande servizio al Re e a Dio-e odiare aquellos que facen al rey o al regno traición, e tra questi sono certo da annoverare i sostenitori dell'Infante de la Cerda che hanno abbandonato Alfonso $X$ per servire il Re di Francia e che erano sul punto di invadere il Regno di Castiglia-León.

Interpretare Qui.m disses come un monito indirizzato ad Alfonso $\mathrm{X}$ affinché il Re si decida a nominare erede il figlio (e a difendere questa scelta di fronte a Filippo l'Ardito) può sembrare lettura azzardata rispetto all'interpretazione vulgata, ma è una lettura coerente con il contesto storico cui allude il vers e che, nonostante l'oscurità di certi passaggi, il vers stesso sembra confermare. La «franqueza y valentía $\rangle^{92}$ che quarant'anni fa Riquer riconosceva alle parole del trovatore narbonese ne esce intatta e rafforzata: non è per mero opportunismo che GrRiq si schiera con i sostenitori di D. Sancho, ma per un forte senso di giustizia che oltrepassa il singolo articolo che regola (in astratto) la successione al trono, per soffermarsi sulle ricadute più ampie (e meno astratte) che avrebbe la scelta del Re. Una scelta (quella di D. Sancho come erede) ugualmente giusta, perché finalizzata al bene del Regno, ma una scelta che Alfonso X ancora non si decide a compiere ${ }^{93}$.

\footnotetext{
${ }^{92}$ Martín de Riquer, Los trovadores, op. cit., III, p. 1621.

${ }^{93}$ Per altro verso, l'interpretazione proposta non è in contraddizione con la complessa struttura che GrRiq ha inteso dare al suo Libre. Qui $\cdot m$ disses è il penultimo vers composto dal trovatore narbonese in terra iberica e, in certo modo, segna il punto di rottura nelle relazioni con il Rei Sabio: GrRiq vi assume -a differenza dei vers precedenti- una prospettiva più contingente alla realtà politica contemporanea -prospettiva segnalata, a livello formale, anche dal fatto che il vers è con tutta probabilità un'imitazione metrica (non un contrafactum, però) della canzone di Guiraudo lo Ros, A la mia fe, Amors-e l'esortazione ad Alfonso X affinché agisca con maggiore risolutezza è forse l'ultimo disperato tentativo del poeta di Narbona di vedersi riconosciuto quello statuto di 'poeta-consigliere' di corte cui ambiva fin dall'inizio del suo soggiorno iberico (cfr. nota 90). Per l'analisi formale e per una più precisa contestualizzazione di Qui $m$ disses nel Libre riquieriano mi permetto di rinviare a Fabio Barberini, «Un'imitazione metrica nel Libre di Guiraut Riquier?», Vox Romanica, 74 (2015), pp. 85-98.
} 
Commentando gli avvenimenti del 1276 (e in modo particolare l'accordo di Vitoria del 7 novembre), Daumet ritiene che quella del Rei Sabio sia stata una vittoria su tutti i fronti:

si l'on considère le fond des choses, il est aisé de reconnaître qu'ils [i francesi] avaient cédé sur tous les points essentiels sans rien recevoir en échange ... Quant à la succession de Castille, Alphonse le Savant gagnait tout, puisque Philippe s'engageait à respecter la décision des Cortès même défavorable à ses neveux ${ }^{94}$.

È un punto di vista molto francese quello di Daumet e Alfonso X avrebbe molto da ridire al riguardo: quello che guadagnò, in fondo, fu solo l'inasprimento delle sue relazioni con la nobiltà castiglianoleonese e soprattutto con il figlio, che di lì a pochi anni avrebbe davvero tentato di spodestare, 'da vivo', il padre ${ }^{95}$. Ma questa è un'altra storia e GrRiq, nel settembre del 1276, non poteva davvero prevederla.

\section{Appendice}

I Qui $\cdot \mathrm{m}$ disses, non a dos ans, que 1 laus me fos desgrazitz del rey n'Anfos, de pretz guitz, 3 mot me fora greus afans; qu'er es tant vil tengutz sa e blasmatz, que sol parlar non aus de luy ad honor, don ai al cor tal dolor, qu'ab pauc chant non desampar. 9

II A moutz homes l'aug blasmar que li foran valedor, si guerra l'agues sabor tant quon a cor de donar. Mas ieu, las, suy en esmay, qu'om me sol lauzar mos chans per elh que m'es abelhitz tant, qu'ieu serai sebelhitz, ans que dreg alhors los lans. 3

6


quar ja gran joy non aurai

tro per ver auia comtar

que $\cdot 1$ sieu enemic maior

aian ab luy tal amor,

que d'elhs no 1 calha gardar.

IV $\mathrm{Ab}$ dreg a volgut renhar ez ab pretz ez ab valor, creyssen de terr'ab lauzor

le reys n'Anfos, que Dieus gar;

ez aras deu mielhs e may voler dreg e patz dos tans, sol que non si'escarnitz, per que de Dieu si'aizitz, e sos pretz no's desenans.

V Mos ditz sera pro bastans, sol que per luy si'auzitz; qu'ieu parli tot esferzitz e si m'enten non l'er dans.

Pero aitant li diray que reys deu amicx amar, mas de l'als dir ai temor: elh chauzisca son melhor, per son dreg dever a far.

VI Jamais no m'esforsarai del rey castellan lauzar, ni d'autre, si en error ven sos pretz, qu'a deshonor me pogues ab dan tornar.

VII No suy astrucx de senhor que $m$ vuelha de cor amar.

(Monica Longobardi, «I vers», art. cit., p. 75)

Recibido: 10/04/2015

Aceptado: 12/01/2016 
$\cos$

\section{QUI·M DISSES, NON A DOS ANS DI GUIRAUT RIQUIER E LA SUCCESSIONE DI ALFONSO X}

RIASSUNTO: Una lunga tradizione critica spiega il vers di Guiraut Riquier, Qui.m disses non a dos ans come commento alla crisi dinastica innescata dalla morte dell'Infante D. Fernando de la Cerda (primogenito di Alfonso $\mathrm{X})$ nel 1275. Quanti si sono occupati del componimento non hanno però chiarito né la posizione di Guiraut Riquier rispetto ai due pretendenti alla corona (D. Alfonso de Cerda, primogenito di D. Fernando, e D. Sancho, secondogenito di Alfonso X), né quale sia il comportamento del Rei sabio che ha provocato tante critiche da parte della corte e del trovatore narbonese. Un'analisi dettagliata del componimento e del contesto storico-politico in cui fu composto porta alla conclusione che Guiraut Riquier rimprovera Alfonso X per l'indecisione mostrata nel riconoscere la successione del figlio D. Sancho e per il poco vigore con cui si stava opponendo alle pretese di Filippo III di Francia (zio di D. Alfonso de la Cerda), che nel settembre del 1276 aveva dichiarato guerra al regno di Castiglia-León per difendere i diritti del nipote.

PARole-Chiave: trovatori, Guiraut Riquier, Alfonso X el Sabio, Sancho IV el Bravo, Regno di Castiglia-León nel Medioevo, storia e storiografia medievale, Siete partidas.

\section{QUI $M$ DISSES, NON A DOS ANS OF GUIRAUT RIQUIER AND THE SUCCESSION OF ALFONSO X}

AbSTRACT: A long tradition of studies reads the vers of Guiraut Riquier, Qui $\cdot m$ disses non a dos ans like a commentary to dynastic crisis aroused after the death of Prince D. Fernando de la Cerda (eldest son of Alfonso X) in 1275. However, the scholars who studied the text don't clarify the position of Guiraut Riquier about the two candidates to the crown (D. Alfonso de la Cerda, the firstborn of D. Fernando, and D. Sancho, second son of Alfonso $\mathrm{X}$ ) and they don't explain what was the behavior of the Learned King which caused so much criticism from the court and troubadour. A detailed analysis of the text and its historical and political context leads to the conclusion that Guiraut Riquier censures Alfonso X by indecision that the king showed to approve the succession of D. Sancho, his son, and by the low strength with which he was facing to claims of Philip III of France (uncle of D. Alfonso de la Cerda) who in September 1276 declared war to the Kingdom of CastillaLeón to defend his nephew's rights.

KeYwords: troubadours, Guiraut Riquier, Alfonso X the Learned, Sancho IV the Brave, Kingdom of Castilla-León in the Middle ages, medieval history and historiography, Siete partidas. 